\title{
Veterans with history of mild traumatic brain injury and posttraumatic stress disorder: Challenges from provider perspective
}

\author{
Nina A. Sayer, PhD; $;^{1-2 *}$ Nancy A. Rettmann, MS; ${ }^{1}$ Kathleen F. Carlson, PhD; ${ }^{1-2}$ Nancy Bernardy, PhD; ${ }^{3}$ \\ Barbara J. Sigford, MD, PhD; ${ }^{1-2}$ Jessica L. Hamblen, PhD; ${ }^{3-4}$ Matthew J. Friedman, MD $^{3-4}$ \\ ${ }^{1}$ Minneapolis Department of Veterans Affairs Medical Center (VAMC), Minneapolis, MN; ${ }^{2}$ University of Minnesota, \\ Twin Cities Campus, Minneapolis, MN; ${ }^{3}$ Executive Division, National Center for Posttraumatic Stress Disorder, \\ VAMC, White River Junction, VT; ${ }^{4}$ Dartmouth Medical School, Hanover, NH
}

\begin{abstract}
The Department of Veterans Affairs (VA) has separate clinical structures and care processes for traumatic brain injury (TBI) and posttraumatic stress disorder (PTSD). However, because veterans are returning from the wars in Iraq and Afghanistan with TBI (most frequently mild TBI [mTBI]) and PTSD, the VA needs to evaluate current service delivery systems. We conducted key informant interviews with 40 providers from across the United States who represented separate clinical teams providing specialized TBI or PTSD services. We identified challenges providers perceive in scheduling and engaging patients with co-occurring mTBI and PTSD (mTBI/ PTSD) in treatment, determining the etiology of patients' presenting problems, coordinating services, and knowing whether or how to alter standard treatments. We found consensus that patients with mTBI/PTSD often have other morbidities requiring specialized treatment, including pain and sleep disturbance. Another important theme we found was the need for patient and family educational material on mTBI/PTSD or pain and mTBI/PTSD and provider education tailored to provider specialty. Together, findings point to the need for guidance for providers on best practices to assess and treat mTBI/PTSD given available information, a systematic approach toward patient and provider education, and research to build the evidence base for practice.
\end{abstract}

Key words: combat disorders, comorbidity, education, health services needs and demands, mild traumatic brain injury, pain, posttraumatic stress disorder, rehabilitation, traumatic brain injury, veterans.

\section{INTRODUCTION}

Each war presents new clinical challenges. Detecting and addressing such challenges often lead to major advances in conceptual understanding, diagnostic assessment, and clinical practice. Today, the current wars in Iraq (Operation Iraqi Freedom [OIF]) and Afghanistan (Operation Enduring Freedom [OEF]) expose U.S. servicemembers to prolonged periods of combat stress and explosive hazards. Consequently, providers in the Department of Veterans Affairs (VA) see patients who may have both

\footnotetext{
Abbreviations: $\mathrm{CPT}=$ cognitive processing therapy, $\mathrm{DOD}=$ Department of Defense, mTBI = mild traumatic brain injury, NCPTSD = National Center for Posttraumatic Stress Disorder, $\mathrm{OEF}=$ Operation Enduring Freedom, OIF $=$ Operation Iraqi Freedom, $\mathrm{PE}=$ prolonged exposure, $\mathrm{PNS}=$ Polytrauma Network Site, PTSD = posttraumatic stress disorder, QI = quality improvement, RAP $=$ Rapid Assessment Process, TBI $=$ traumatic brain injury, VA = Department of Veterans Affairs, VAMC $=$ VA medical center, VISN $=$ Veterans Integrated Service Network.

*Address all correspondence to Nina A. Sayer, PhD; Center for Chronic Disease Outcomes Research, VA Medical Center, One Veterans Drive (152/2E), Minneapolis, MN 55417; 612-467-4623; fax: 612-727-5699.

Email: nina.sayer@va.gov

DOI:10.1682/JRRD.2009.01.0008
} 
combat-related stress disorders and explosive-induced concussive injury. In this article, we report the results of interviews with VA providers faced with the challenge of treating these veterans.

In this era of modern warfare, combat involves exposure to blasts caused by improvised explosive devices, rocket-propelled grenades, and other explosives. Blast injuries are often polytraumatic, meaning that they affect more than one body region or system [1]. Particularly common is traumatic brain injury (TBI). It has been estimated that over 60 percent of blast injuries result in traumatic brain injury (TBI) [2-3]. Because of its frequency relative to that observed in earlier U.S. conflicts [4], TBI has been labeled the "signature injury" in the Global War on Terror [5].

The prevalence of TBI among OIF/OEF servicemembers is difficult to ascertain, particularly because providers may miss less-severe forms of TBI or mistakenly label other disorders as TBI. However, recent research suggests that the prevalence may approach 20 percent, with the majority of TBI cases among OIF/ OEF returnees being mild in severity [6-7]. The American Congress of Rehabilitation Medicine has defined mild TBI (mTBI) as a physiological disruption of brain function as manifested by at least one of the following: alteration of mental state, loss of consciousness, loss of memory or focal neurological deficit, that may or may not be transient; but where the severity of the injury does not exceed the following: posttraumatic amnesia for greater than 24 hours, after the first 30 minutes Glasgow Coma Score 13 to 15, and loss of consciousness is less than 30 minutes [8]. Generally, the terms mTBI and concussion are used interchangeably.

Available research suggests that although the majority of mTBI cases resolve within weeks or months, some individuals develop persistent postconcussive symptoms [9]. These symptoms can be categorized as physical, cognitive, and behavioral/emotional. Physical symptoms include headaches, dizziness, fatigue, and disordered sleep; cognitive symptoms include deficits in concentration and memory; and behavioral/emotional symptoms include irritability, depression, and emotional lability [10-11]. Most postconcussive symptoms are nonspecific and reported in individuals with no history of head trauma who have other conditions, such as chronic pain and psychiatric disorders [12]. Although a variety of symptoms associated with TBI may occur, symptom manifestation is not part of the definition [11].
The current wars in Iraq and Afghanistan are also associated with a high burden of psychiatric disorders, with posttraumatic stress disorder (PTSD) being the most common [13]. PTSD is an anxiety disorder that follows exposure to life-threatening experiences such as war, sexual assault, homicide, motor vehicle crashes, and natural disasters. The sufferer vacillates between the intense distress associated with unwanted trauma-related memories and psychic numbness. Also present is hypervigilance, sleep disturbance, and other manifestations of physiological arousal [14]. Recent reports indicate that the rate of PTSD and other psychiatric disorders in returning OIF/ OEF servicemembers is similar to the rate of TBI and that 37 to 44 percent of those with possible mTBI may also have PTSD and/or depression [6-7].

The VA is charged with meeting the needs of veterans returning from OIF/OEF with combat-related emotional and physical wounds. Since 1989, the VA has operated specialized inpatient and outpatient clinics to treat PTSD, and it is now the largest provider of PTSD services in the United States. Nationally, the VA offers 163 specialized PTSD programs; most are outpatient PTSD teams $(n=112)$. Veterans who screen positive for PTSD on the postdeployment mental health screening are often referred to PTSD teams for evaluation and treatment [15]. Cognitive therapy, exposure therapy, stress inoculation training, and eye movement desensitization and reprocessing are strongly recommended for treatment of PTSD (Department of Defense [DOD] and VA guidelines for PTSD [16]). Within the VA, the National Center for PTSD (NCPTSD) is currently leading national rollouts of cognitive processing therapy (CPT) and prolonged exposure (PE), the most effective evidence-based psychotherapies for PTSD. Recommendations for pharmacotherapy are detailed in the PTSD clinical practice guidelines [16].

In 2005, the VA developed the "Polytrauma System of Care" to rehabilitate OIF/OEF veterans with TBI and other physical war-related injuries. The Polytrauma System of Care consists of the following four components: (1) specialized regional inpatient rehabilitation centers accredited in TBI; (2) specialized outpatient and subacute rehabilitation programs geographically distributed within each of the 21 Veterans Integrated Service Networks (VISNs); (3) designated polytrauma teams at smaller, more remote VA facilities; and (4) a point of contact at all other VA facilities. The specialized outpatient and subacute rehabilitation programs are called Polytrauma Network 
Sites (PNSs) and manage the postacute sequelae of combat injuries, including TBI, and coordinate lifelong rehabilitation services for veterans with polytrauma or blastrelated injuries living in their catchment area [17]. At the time of this research, 21 PNS sites existed. Subsequently, the VA added another PNS site in Puerto Rico. Patients who screen positive for TBI on the VA postdeployment TBI Screening Clinical Reminder are referred to polytrauma teams for follow-up evaluation and treatment [18]. Treatment of mTBI generally involves patient and family education and, if symptoms persist, management of specific symptoms. The VA recently released Clinical Practice Guidelines for Management of Concussion/Mild Traumatic Brain Injury [19]. The clinical practice guidelines present separate algorithms for initial presentation for treatment, the management of symptoms related to mTBI, and follow-up of persistent symptoms.

The VA-specialized PTSD programs and the Polytrauma System of Care have separate developmental histories and administrative structures. The VA did not design them specifically for patients with problems consequent to both physical and psychological trauma. Furthermore, little to no data currently exist to guide the VA in the management of these patients. The challenges providers face to meet the needs of OIF/OEF veterans who have both a history of mTBI and PTSD (mTBI/PTSD) in the absence of an evidence base for practice have not been examined previously. Such information is needed to identify opportunities for improvement and priorities for research. To begin to fill this gap, we gathered preliminary data from VA providers that could be used to inform systemwide efforts to improve services offered to OIF/ OEF veterans with mTBI/PTSD. We focused on providers working within PNS and PTSD teams because it is likely that these teams have the highest concentration of veterans with $\mathrm{mTBI} / \mathrm{PTSD}$ and because they have leadership roles within the VA in defining best practice for $\mathrm{mTBI}$ and combat stress, respectively.

\section{METHODS}

\section{Design}

We developed this project in collaboration with the VA Department of Physical Medicine and Rehabilitation and the NCPTSD as a quality improvement (QI) project to meet the need for information to improve practice for veterans with mTBI/PTSD. The Minneapolis VA Medical
Center (VAMC) Institutional Review Board designated this project as QI.

We conducted semistructured interviews with providers working within either PTSD programs or PNSs to understand their view of challenges providing medical care to OIF/OEF veterans with mTBI/PTSD. Consistent with Rapid Assessment Process (RAP) methodology, we used multiple interviewers and iterative data collection and analysis to rapidly gather as much data as possible and reach content saturation in key areas [20-21]. Unlike typical RAP studies, however, we did not use a field liaison. A field liaison is a member of the group being studied who can help the research team understand the fieldwork site and provide logistical support. We did not use a field liaison for this project because we interviewed providers across the country by telephone, eliminating the need for and feasibility of on-site logistical support. In addition, the research team consisted of researchers who collaborate with polytrauma and PTSD teams, reducing our need for an insider's assistance to interpret informants' responses.

\section{Recruitment Methods and Participants}

Staff with leadership roles within the NCPTSD and the VA Department of Physical Medicine and Rehabilitation each identified one representative with specialized knowledge about team practices for OIF/OEF veterans with mTBI/PTSD within each of the VA's 21 service areas. The NCPTSD and the VA Department of Physical Medicine and Rehabilitation Program Office selected providers who were points of contact for dissemination of information on PTSD or TBI within their respective VISNs. The research team emailed recruitment materials to these providers. Recruitment materials included a cover letter describing the project and a list of interview questions. The recipient was asked to contact a designated member of the study team for scheduling purposes if willing to volunteer and/or to discuss the project with their team to see if someone else could represent the team. A member of the research team emailed each volunteer clinician a brief survey asking about the clinician's role on the team, number of years working within VA, and the team's experience treating OIF/OEF veterans with mTBI/PTSD. In addition, we emailed volunteers the interview questions and asked them to discuss the questions with their team before the interview so they could represent their team during the interview. Of the 42 potential volunteers (21 PTSD team members and 21 PNS 
team members), 40 underwent the interview. The remaining two did not respond.

Table 1 lists participants' professional titles and years working at the VA. Fourteen of the PTSD and five of the PNS providers described themselves as having administrative leadership roles within their teams or departments, including program director or coordinator $(n=10)$, service or section chief $(n=2)$, medical director $(n=3)$, and other $(n=4)$. To protect the anonymity of the informants, we do not report potentially identifying professional titles, demographics, or facility-related information. Participants came from 32 VAMCs spread across the 21 VISNs; eight PNS and PTSD informants worked in the same facility. As can be seen in Table 1, most informants were psychologists or neuropsychologists. This is not surprising given that psychologists and neu- ropsychologists typically assess and treat veterans with psychiatric disorders and TBI.

\section{Interview Content}

The research team developed semistructured interview guides to elicit information on assessment and treatment needs of patients with mTBI/PTSD. We developed one interview guide for PNS providers and a parallel guide for PTSD team providers. Table 2 lists the interview topics and illustrative questions. Although we covered all topics during each interview, we did not necessarily ask questions using the same wording or in the same order. This process allowed us to follow the natural flow of the dialogue. We refined the interview guides during the first few interviews [22-23].

Table 1.

Professional characteristics of Department of Veterans Affairs (VA) provider participants $(N=40)$.

\begin{tabular}{lcc}
\multicolumn{1}{c}{ Characteristic } & $\begin{array}{c}\text { PTSD Team Members } \\
(\boldsymbol{n}=\mathbf{2 1})\end{array}$ & $\begin{array}{c}\text { PNS Team Members } \\
(\boldsymbol{n}=\mathbf{1 9})\end{array}$ \\
Title & 18 & 7 \\
$\quad$ Psychologist & 0 & 10 \\
$\quad$ Neuropsychologist & 3 & 0 \\
$\quad$ Psychiatrist & 0 & 1 \\
$\quad$ Physiatrist & 0 & 1 \\
$\quad$ Advanced Registered Nurse & $8.32 \pm 6.02$ & $7.55 \pm 6.92$ \\
Years Working at VA (mean \pm standard deviation) & \\
PNS = Polytrauma Network Site, PTSD = posttraumatic stress disorder.
\end{tabular}

Table 2.

Interview topics and illustrative questions for Polytrauma Network Site and posttraumatic stress disorder (PTSD) team providers.

\begin{tabular}{ll}
\hline \multicolumn{1}{c}{ Topic } & \multicolumn{1}{c}{ Questions } \\
\hline Referral Process & What is process by which patients come to your clinic? \\
& Is this process different for patients who have symptoms of PTSD and \\
TBI? & How are patients evaluated in your clinic? \\
Assessment and Challenges & $\begin{array}{l}\text { Does your clinic alter this evaluation process because of presence of } \\
\text { [PTSD or TBI]? }\end{array}$ \\
Treatment and Challenges & $\begin{array}{l}\text { Has your clinic modified treatment approaches or developed new } \\
\text { treatment approaches because of presence of [PTSD or TBI]? }\end{array}$ \\
Cross-Team Collaboration & $\begin{array}{l}\text { How is your team working with other services in your facility to } \\
\text { assess/treat patients with a history of both PTSD and TBI? }\end{array}$ \\
Other Comorbidities and Problems & What other problems or issues are particularly prevalent or clinically \\
important in this population? & What terms do you use to talk to patients about their TBI and PTSD? \\
Terminology & Is there consensus on terminology on your team? \\
& What is needed to treat patients with PTSD and TBI more effectively \\
\end{tabular}




\section{Data Collection}

The interview team consisted of three master-level and two doctoral-level researchers. Two members of the interview team participated in each interview: one conducted the interview and the other took detailed notes on a computer and asked clarifying questions at the end of the interview. We rotated these roles across interviews. Following each interview, the two interview team members debriefed and wrote a conjoint interview log (see "Analysis" section) that we circulated among all members of the research team so that all members were aware of emergent themes. We conducted interviews over the telephone that lasted approximately 30 minutes. The team conducted the interviews between July 2 and October 3, 2008. When indicated, a member of the research team contacted the volunteer through email after the interview to request examples of educational material that the volunteer had discussed using and/or to ask clarifying questions.

\section{Analysis}

The analysis included a consensus-seeking discussion among the interviewers right after each interview. The product of this discussion was a postinterview log that summarized the volunteer's responses to each question, the major themes, and new content areas for further inquiry in future interviews $[21,24]$. We then developed a summary of findings across interviews through constant comparison and interview team consensus. We circulated the summary to participants for feedback, which we then used to refine the findings further. The document included a one-page summary of the purpose and methods of this project, as well as a draft version of the results that was highly similar to Figures 1 and 2.

\section{RESULTS}

We organized findings by interview and emergent topics. Figure 1 summarizes challenges PNS and PTSD providers identified from screening and referral through treatment, as well as gaps with respect to services for behavioral and health problems other than PTSD and TBI. The themes within each topic area come from providers across teams and VISNs and represent the scope of challenges VA PTSD and PNS providers face in their efforts to deliver services to OIF/OEF veterans with mTBI/PTSD.

\section{Screening and Referral}

According to our informants, OIF/OEF patients are generally referred to PTSD and PNS clinics for assessment and/or treatment after screening positive for PTSD or TBI in other settings, such as primary care or clinics developed specifically for OIF/OEF veterans. Concern existed among a few PNS and PTSD providers that primary care providers and residents may not know how to probe for mTBI and that, consequently, some mTBI sufferers may go undetected. Another concern was positive screens-some providers believed that patients may not understand why they are being referred to TBI and PTSD specialty clinics following positive screening for TBI and PTSD. PNS providers brought up this problem more frequently regarding positive TBI screens. One PNS provider suggested that VA providers conducting TBI screening may give veterans who screen positive for TBI the inaccurate impression that the positive screening result indicates that they have a brain injury.

\section{Assessment}

Most PTSD and PNS teams perform an intake process and conduct assessments, although a few clinics focus exclusively on treatment. PNS teams often assess for PTSD using interviews and self-report questionnaires (e.g., PTSD Checklist [25]); most PTSD teams do not formally assess TBI, although they may complete the 4-item VA TBI screening tool [18]. There was remarkable consensus across providers in PNS and PTSD teams with regard to challenges of determining whether a patient's current symptoms result from mTBI or PTSD or both. This is because of the overlap in symptoms associated with a concussion and PTSD. Some PNS providers explained that their teams focus on their patients' symptoms and functional problems rather than etiology. The comments made by a few PNS and several PTSD providers suggested that some providers mistakenly believed that the diagnosis of TBI depends on a patient reporting current postconcussive symptoms (e.g., concentration problems), just as a diagnosis of PTSD depends on the patient reporting current intrusive, avoidant, or hyperarousal symptoms. We did not, however, formally assess provider knowledge of the definition of mTBI.

Providers believed that assessment of patients with mTBI/PTSD takes longer and requires a high level of skill. One PNS clinician stated, "It [the presence of PTSD] complicates and lengthens the assessment process for physicians and psychologists and others. The issue of 


\section{Screening and Referral}

1. Potential for false negative TBI screening results if providers not trained to probe for TBI.

2. Potential for patient confusion about screening and evaluation processes, particularly for TBI.

\section{Assessment}

1. Difficulties making definitive diagnosis of $\mathrm{mTBI}$ because of duration of time between injury and VA assessment and lack of access to DOD records.

2. Difficulty determining whether patients' functional problems and symptoms are related to history of mTBI, PTSD, or both.

3. Clinically complex patients presenting with comorbidities and clinical problems in addition to mTBI/PTSD, including pain, sleep problems, depression, and substance use disorders.

4. Uncertainty as to whether findings from research on sport-related concussion are transferable to blast-related mTBI.

5. Uncertainty as to whether traditional cutoff scores on psychological testing (e.g., scores to determine exaggeration) are applicable to individuals with blast-related TBI.

6. Redundancies in assessments conducted by PTSD and PNS teams.

7. Secondary gain issues possibly influencing symptom reporting.

\section{Scheduling and Patient Engagement}

1. High rates of no-shows.

2. Lack of systems to consolidate and coordinate appointments and medications across providers and clinics.

3. Lack of specialty services in rural areas of United States.

4. Logistical barriers to treatment, including lack of time and money to travel and attend appointments.

5. Families facing same logistical barriers to treatment involvement as veteran patients.

\section{Treatment}

1. Uncertainty as to whether and how to alter evidence-based treatment for PTSD when patient has history of mTBI.

2. Lack of protocols (e.g., skills training) specifically for mTBI/PTSD.

3. Patients with $\mathrm{mTBI} / \mathrm{PTSD}$ requiring more individual attention, repetition, and time to complete assignments and benefit from PTSD treatment.

4. Rehabilitation providers needing to slow pace and incorporate emotion-management into interventions for symptoms associated with mTBI.

5. Psychiatrist prescribing medications contraindicated for TBI; physiatrists prescribing medications contraindicated for PTSD.

6. Provider stress because of larger caseloads, new responsibilities and, in some cases, understaffed teams.

7. Lack of dedicated psychiatrist time on most PNS teams.

\section{Collaboration and Coordination of Care}

1. Coordination/collaboration across PNS and PTSD teams taking additional clinician initiative and time.

2. Geographic distance between teams interfering with communication.

3. Uncertainty as to how and whether to sequence treatment for persistent symptoms associated with mTBI and PTSD.

4. Differences between mental health and rehabilitation models of care.

5. Patients with $\mathrm{mTBI} / \mathrm{PTSD}$ receiving services through multiple teams may receive medications that are contraindicated for one or more of their conditions or disorders.

\section{Terminology and Labeling}

1. Patients with inaccurate and countertherapeutic understanding of positive TBI screen and mTBI diagnosis.

2. Inconsistencies in terminology providers use to describe TBI confuse patients and families.

3. Patients preferring to have diagnosis of TBI instead of diagnosis of PTSD because of stigma associated with PTSD.

4. Term "polytrauma" confusing and upsetting patients.

5. Patients with anxiety about having both PTSD and mTBI.

\section{Services for Other Behavioral and Health Problems}

1. Lack of availability of pain and sleep disorder expertise in many VA facilities and U.S. regions.

2. Need for more and tailored vocational services for OIF/OEF veterans.

3. Need for family services and couples therapy.

Figure 1.

Challenges in treating Operation Iraqi Freedom and Operation Enduring Freedom (OIF/OEF) veterans with history of mild traumatic brain injury $(\mathrm{mTBI})$ and posttraumatic stress disorder $(\mathrm{PTSD}) . \mathrm{DOD}=$ Department of Defense, PNS = Polytrauma Network Site, TBI = traumatic brain injury, VA = Department of Veterans Affairs. 
what to treat first always comes up due to the multiplicity of problems." Providers who had experience treating civilians with a history of mTBI stated that OIF/OEF veterans with mTBI are clinically more complex, particularly regarding the prevalence of psychiatric comorbidities. Providers believed that research on the cumulative effects of blast exposure might explain why postconcussive symptoms seem to persist longer in OIF/OEF veterans with a history of mTBI. These clinicians wondered if research based on civilians with a history of mTBI (e.g., sport-related concussion) generalizes to those who have been exposed to multiple blasts.

\section{Scheduling and Patient Engagement}

PNS and PTSD providers described perceived problems associated with scheduling patients for assessment and treatment. Patients may be difficult to contact for scheduling, no-show their appointments, or find themselves on long clinic waiting lists. Sometimes patients were referred to both PNS and PTSD teams at the same time, and according to the providers we interviewed, there was no system for coordinating appointments between the teams.

Appointment no-shows were felt to be particularly high in OIF/OEF veterans referred to the PTSD and PNS clinics. Providers expressed uncertainty as to whether the pattern of no-shows is particular to patients with TBI, PTSD, mTBI/PTSD, or an OIF/OEF cohort effect. For example, some providers wondered whether PTSDrelated problems with anger, trust, being around other people, and/or mTBI-related problems with memory and attention interfere with attendance. Several informants stated that these patients need more and different types of appointment reminders than are typically given. For instance, a few providers stated that their team uses more frequent telephone calls and letters to remind OIF/OEF patients to attend appointments than they use with other veteran cohorts. Some providers expressed concern that OIF/OEF veterans have more medical appointments in general compared with patients of other service eras and discussed the need to consolidate and coordinate care across these appointments. One provider expressed concern that the number of appointments among OIF/OEF veterans was producing "professional patients" rather than promoting recovery.

Providers brought up logistical barriers to patient participation in treatment, including lack of access to services for rural veterans, lack of time to travel and attend appointments because of work and childcare responsibilities, and financial constraints. The same problems existed regarding family member involvement in treatment.

\section{Treatment}

Most informants described patients being treated for PTSD by the local PTSD team. However, PNS providers are becoming educated about PTSD, and several PNS teams provide both PTSD treatment and rehabilitation to at least some OIF/OEF patients with mTBI/PTSD. Across teams, a lack of consensus existed regarding whether or how to alter PTSD treatment strategies for patients who have a history of mTBI. Several informants stated that their teams use standard evidence-based PTSD treatments. Other teams reported using parts of standard protocols, such as the cognitive component of CPT, or implementing standard protocols with "more flexibility" (e.g., by altering expectations for homework completion and attendance and engaging family member support). Some PTSD providers believed that PE is associated with better treatment participation and outcomes compared with CPT "because it is not so heavily reliant on memory."

\footnotetext{
- Patient educational material on mTBI/PTSD or triad of PTSD, mTBI, and pain.

- Awareness of existing educational material through VA and DOD for providers.

- Good understanding of TBI, particularly mTBI, or of how rehabilitation works for providers.

- Education for rehabilitation specialists working with psychiatric patients (e.g., responding to suicidality and patient anger or aggression).

- Education on medications that are contraindicated for TBI or PTSD for physicians.

- Education on cognitive rehabilitation and best practices for mTBI for mental health staff.

- Education on evidence-based psychotherapies for PTSD for PNS staff.
}

Figure 2.

Patient and provider educational needs. DOD = Department of Defense, mTBI = mild traumatic brain injury, PNS = Polytrauma Network Site, PTSD = posttraumatic stress disorder, TBI = traumatic brain injury, VA = Department of Veterans Affairs. 
Many providers believed that patients with mTBI require more individual attention, repetition, and time to complete assignments and to benefit from PTSD interventions. As one PTSD clinician stated, "The presence of TBI makes everything go much more slowly. There is a lot more repetition.” Consensus existed regarding the need for research to evaluate the effect of mTBI on the efficacy of the evidence-based PTSD treatments being disseminated throughout the VA.

PNS teams treat the symptoms associated with mTBI in patients with mTBI/PTSD. PNS providers described slowing the pace and incorporating emotion management techniques and/or education on PTSD and associated problems, such as depression and substance use disorders, into skills training and educational interventions. Some PNS teams have developed or are in the process of developing new psychoeducational groups specifically for patients with mTBI/PTSD. One PNS team developed a program for OIF/OEF patients with PTSD, TBI, and pain. Several PNS providers explained their view that rehabilitation interventions targeting functional problems or cognitive symptoms can also help patients manage their PTSD. For example, one PNS provider believed that PTSD-related symptoms may improve when patients learn skills during occupational therapy to better organize their home environment or during recreational therapy to engage in activities outside of home.

PNS and PTSD providers brought up the challenge of determining how or whether to sequence PTSD treatment and treatment for symptoms associated with mTBI. Some teams prefer that patients undergo PTSD treatment first, other teams sequence treatment so that the more dominant or distressing problem is addressed first, and some teams or individual providers prefer to treat both problems at the same time. Providers from both teams also discussed the potential value of a truly integrated PNSPTSD team or at least of having a representative from the PTSD team work on the PNS team and vice versa.

\section{Collaboration and Care Coordination}

The interview data suggested considerable variation with regard to the degree and type of collaboration between PTSD and PNS teams across VISNs. According to those we interviewed, close or effective collaboration existed in some facilities and less close or developing collaboration existed in other facilities. Several providers talked about improvements in care coordination and collaboration across teams to meet the needs of OIF/OEF patients with mTBI/PTSD.

The research team's impression was that coordinating assessment and treatment services depended on individual clinician initiative and could take considerable time. As one provider explained, "It is up to the provider to reach out, which can be challenging when both [PTSD and PNS] providers have full schedules.” Geographic separation between teams can create an added challenge to collaboration. Two providers explicitly referred to differences between the mental health and rehabilitation approaches toward treatment as interfering with effective collaboration. One belief was that rehabilitation providers expect a more rapid recovery than mental health providers who were described as accustomed to slow change and chronic illness. Another belief was that rehabilitation providers more aggressively follow up with patients, whereas mental health providers expect patients to be responsible for keeping appointments and completing homework.

Many PNS providers talked about potential problems managing medications across the teams and care settings. One particular concern was that physicians outside of rehabilitation may prescribe medications that are contraindicated for TBI. We identified only two PNS teams with dedicated psychiatry time for patients, although several other teams noted that this would be helpful. A PTSD team clinician also brought up the concern that physiatrists may prescribe medications inappropriate for PTSD.

\section{Terminology and Diagnostic Labels}

A lack of consensus existed across PNS and PTSD teams, and sometimes within teams, around terminology to use when discussing mTBI with patients. Many PNS and some PTSD teams preferred to use the term "concussion" instead of mTBI because they believed the label concussion promotes hope in recovery compared with the labels TBI or "brain injury." In contrast, other PNS and most PTSD teams preferred to use the term TBI, believing that concussion could be construed as a euphemism and that patients are familiar with the label TBI through the military, publicly available information, and the VA disability benefit system. Less variation existed regarding the label for PTSD, but some PNS teams used terms like "battle stress," "battle mind," or "combat stress" instead of or in addition to PTSD. Across PNS and PTSD teams, strong consensus existed regarding the importance of 
emphasizing recovery and educating patients about the meaning of the diagnostic labels. Some providers were concerned about confusing patients through use of multiple terms across providers and settings.

Many providers believed that patients preferred a diagnosis of TBI instead of a diagnosis of PTSD because of the stigma associated with PTSD. A PNS informant explained, "Patients are really struggling with how to label their condition. They prefer attributing it to TBI because they don't want to be labeled as "crazy."” Some PNS providers said that they "lose" patients when they refer them to the PTSD team for treatment or when patients see that they have an appointment scheduled with a psychologist. Several PTSD and PNS providers seemed to believe that it is hard for patients to understand prognosis and to cope emotionally when told that they have both diagnoses. Another clinician expressed the concern that the term polytrauma "stuns" patients, meaning that it frightens and confuses them.

\section{Other Comorbidities and Service Needs}

Many providers emphasized that mTBI/PTSD also co-occur with other problems requiring clinical attention, including pain (head and musculoskeletal), sleep disturbance, substance use disorders, and depression. They expressed particular concern about the lack of availability of adequate treatment for pain and sleep-related problems. One PNS clinician explained that veterans have to wait several months to obtain VA treatment for headache and sleep problems, saying, "If the headaches are not being treated, it is hard to work on cognitive rehabilitation. Community pain and sleep clinics are not available." Across interviews, headache pain was the most commonly described co-occurring problem warranting clinical attention. Clinicians also brought up psychosis; attentiondeficit/hyperactivity disorder; and audiological, vision, and vestibular problems as significant concerns in OIF/ OEF veterans with mTBI/PTSD but with less frequency.

Several providers brought up employment difficulties as a significant problem for this group of younger veterans and discussed the need for more and improved vocational services. They also discussed family interventions (e.g., families and couples therapy and parenting classes) as a need among OIF/OEF veterans with mTBI/ PTSD.

\section{Patient and Provider Educational Needs}

Figure 2 lists educational needs providers discussed. Most teams distributed separate information on PTSD and/or TBI to patients and families rather than educational material that discusses mTBI/PTSD. Some teams have been developing their own material. They expressed concern about the existing material on TBI or PTSD being dry and wordy. Some informants seemed unaware of educational material on TBI or PTSD available through national resources, including the DOD. Providers from both PNS and PTSD teams across regions described the need for educational materials for patients and families on co-occurring PTSD and persistent symptoms associated with TBI. Some expressed the need for material that focuses on the triad of PTSD, TBI, and pain.

Providers also identified provider educational needs. One PNS clinician explained, "Provider education is a must. This would help to ensure that veterans will receive a consistent message about the potential impact of their history of TBI on their current functioning and its association with PTSD.” Many providers emphasized that VA providers, particularly those outside of rehabilitation settings, may lack a good understanding of TBI and rehabilitation. A specific concern was that VA providers may misattribute all the patient's current symptoms, including mental health problems, to TBI and believe that a TBI specialist is needed whenever a patient carries a TBI diagnosis when this is not the case. Conversely, some providers expressed concern about persistent symptoms associated with mTBI being misattributed to psychiatric disturbance when the patient may benefit from education on mTBI. Providers also discussed the importance of training rehabilitation providers to provide evidencebased treatment for PTSD and to work with psychiatrically distressed (e.g., suicidal or aggressive) patients.

\section{DISCUSSION}

OIF/OEF patients with mTBI/PTSD have clinical problems that traverse traditional team boundaries (e.g., rehabilitation and mental health service lines). The providers we interviewed described a lack of systems to coordinate appointments, services, and medications across teams. Apparently, individual providers must review chart notes, medications, and scheduling information to coordinate service delivery. In light of the frequency with which patients are seen by both PTSD and 
rehabilitation providers, the VA may need to consider models of care that more closely integrate these traditionally distinct services. In addition, providers emphasized that patients with mTBI/PTSD often have other morbidities requiring specialized treatment, such as pain, sleep disturbance, and substance use disorders. Providers emphasized these problems because they affect a patient's ability to engage in treatments. Models of care that depend on consultation with specialists rather than integrative care may be insufficient for OIF/OEF veterans with deployment-related comorbidities. These findings are consistent with prior observations of OIF/OEF veterans with multiple morbidities [26]. A few providers also brought up their views on differences between the rehabilitation and mental health teams' understanding and approach toward patients. This topic warrants systematic exploration because such differences may confuse patients and interfere with cross-team collaboration.

Providers also believed that OIF/OEF patients are difficult to engage and frequently do not show up for appointments. Anecdotally, this perception is widely held among VA providers; however, we know of no published empirical studies on this issue. Research on patient engagement among OIF/OEF veterans is needed. It would be important to determine whether common strategies such as integrated practice models and scheduling evening and weekend clinics improve patient engagement and appointment adherence. The VA should also determine whether telemedicine options, which may overcome some of the identified barriers to appointment adherence, are being utilized sufficiently.

The theme of clinician uncertainty runs through many of the challenges providers discussed. In particular, there was uncertainty as to patients' reactions to diagnostic labels, the etiology of patients' presenting problems, whether to treat PTSD and symptoms associated with mTBI concurrently or sequentially, and whether or how to alter standard treatments for PTSD. This uncertainty is understandable in light of known difficulties disentangling physical from emotional etiology and the lack of health services and treatment outcome studies focused on mTBI/PTSD [27]. Most scientific literature on co-occurring PTSD and TBI has focused on the controversy as to whether PTSD can occur after a TBI in which amnesia occurs [28-33].

At present, teams appear to be using different VA and DOD patient educational material. Some teams are even creating their own educational material because they could not identify resources that appear to meet the needs of OIF/OEF patients and their families. Review and dissemination of the available and developing materials would fill an immediate need in the field.

Informants also described gaps in provider education. Findings based on these brief interviews suggest that the educational needs of primary care providers, mental health professionals, and rehabilitation specialists may not be the same and, therefore, that the VA will need to tailor its educational strategies for different provider groups. Comments made during the interviews suggested that some providers mistakenly believed that a TBI diagnosis is based on evaluation of current symptoms associated with a head trauma. Since collection of these data, the VA has used satellite broadcasts to educate providers on the criteria for diagnosing TBI [34]. Whether this educational effort has corrected this misconception remains unknown. A formal evaluation of educational needs among providers who provide services to patients with mTBI may help the VA plan and evaluate future trainings.

They are limitations to this QI project. First, findings are based on self-report during a brief one-time interview and may be influenced by various types of self-report and memory biases. Although the interview team explained procedures to protect anonymity, some participants may have been reluctant to voice their concerns. We obtained information about provider perceptions and did not measure actual behavior or the capacity of the system to meet the needs of OIF/OEF veterans with mTBI/PTSD. Research studies that build on this QI project should consider using site visits, observations of service delivery, administrative data, standardized questionnaires, and patient assessments to understand care processes and outcomes more fully. Second, although we asked volunteers to discuss the interview questions with their team and be prepared to represent their whole team during the interview, we do not know whether the perspective of informants represented other providers and professional disciplines on his or her team. A related limitation is that we sampled providers working in 32 VAMCs spread across the 21 VISNs. This sample represents only a small percent of the sites where veterans with $\mathrm{mTBI} / \mathrm{PTSD}$ receive care, and findings may not generalize across all VAMC sites treating $\mathrm{mTBI} / \mathrm{PTSD}$. Inclusion of methods to collect data from more individuals at each site and from more sites would have strengthened the conclusions of this project. Last, the structures and processes of care in place for assessment and treatment of OIF/OEF veterans in 
general and OIF/OEF veterans with TBI in particular are rapidly evolving. More staff are being hired, new clinics are being established, and staff trainings are in various stages of planning and execution. Findings may therefore be time-dependent. We can, however, use them as a baseline against which to measure change as the VA continues to expand and improve services for OIF/OEF veterans with war-related comorbidities.

\section{CONCLUSIONS}

Providers reported that OIF/OEF veterans returning with war-related TBI, PTSD, and other comorbidities present challenges for the VA's existing care structures and treatment approaches. The VA is forging new ground as it responds to the needs of OIF/OEF veterans with blast-related physical injuries and traumatic stress. Taken together, this QI project points to the need for guidance to providers on best practices to assess and treat mTBI/ PTSD given available information, a systematic approach toward patient and provider education, and research to build the evidence base for practice in this area. Providers' concerns about care coordination, labeling, and patient engagement suggest a need for research focused on the healthcare delivery system and patient expectations and preferences, as well as on interventions.

\section{ACKNOWLEDGMENTS}

\section{Author Contributions:}

Study concept and design: N. A. Sayer, N. A. Rettmann, K. F. Carlson, N. Bernardy, B. J. Sigford, J. L. Hamblen, M. J. Friedman. Acquisition, analysis, and interpretation of data: N. A. Sayer, N. A. Rettmann, K. F. Carlson, N. Bernardy, B. J. Sigford, J. L. Hamblen, M. J. Friedman.

Drafting of manuscript: N. A. Sayer, N. A. Rettmann, K. F. Carlson, N. Bernardy, B. J. Sigford, J. L. Hamblen, M. J. Friedman. Critical revision of manuscript for important intellectual content: N. A. Sayer, N. A. Rettmann, K. F. Carlson, N. Bernardy, B. J. Sigford, J. L. Hamblen, M. J. Friedman.

Obtained funding: N. A. Sayer.

Study supervision: N. A. Sayer.

Financial Disclosures: The authors have declared that no competing interests exist.

Funding/Support: This material was based on work supported by a VA Health Services Research and Development Quality Enhancement Research Initiative Locally Initiated Project (grant PLY 08-05-3).

Additional Contributions: We would like to thank the PTSD and PNS team representatives who participated in this project.
Participant Follow-Up: The authors do not plan to inform participants of the publication of this study. However, the authors will help disseminate this article to VA providers who treat veterans with mTBI and PTSD.

\section{REFERENCES}

1. Veterans Health Administration. Polytrauma rehabilitation centers [Internet]. Washington (DC): Department of Veterans Affairs; c2005. VHA Directive 2005-024; 2005 Jun 8 [cited 2009 Jun 25]; [about 11 screens]. Available from: http://www1.va.gov/vhapublications/ViewPublication.asp?pub ID $=1274$

2. Okie S. Traumatic brain injury in the war zone. New Engl $\mathrm{J}$ Med. 2005;352(20):2043-47. [PMID: 15901856] DOI:10.1056/NEJMp058102

3. Warden DL, Ryan LM, Helmick KM, Schwab K, French L, Lu W, Lux WE, Ecklund J, Ling F. War neurotrauma: The Defense and Veterans Brain Injury Center (DVBIC) experience at Walter Reed Army Medical Center. J Neurotrauma. 2005;22:1178.

4. Warden DL. Military TBI during the Iraq and Afghanistan wars. J Head Trauma Rehabil. 2006;21(5):398-402.

[PMID: 16983225]

DOI:10.1097/00001199-200609000-00004

5. Newsweek: The war in Iraq [Internet]. New York (NY): Newsweek; c2009. Riccitiello R. Iraq. A marine's experience of brain injury. Damaged brains are emerging as the singular injury of the Iraq conflict. A soldier's story; 2007 Mar 17 [cited 2009 Jun 25]; [about 4 screens]. Available from: http://www.msnbc.msn.com/id/11882164/site/newsweek/displaymode/1098/.

6. Tanielian TL, Jaycox L, editors. Invisible wounds of war: Psychological and cognitive injuries, their consequences, and services to assist recovery. Santa Monica (CA): RAND; 2008.

7. Hoge CW, McGurk D, Thomas JL, Cox AL, Engel CC, Castro CA. Mild traumatic brain injury in U.S. soldiers returning from Iraq. N Engl J Med. 2008;358(5):453-63. [PMID: 18234750] DOI:10.1056/NEJMoa072972

8. American Congress of Rehabilitation Medicine. Definition of mild traumatic head injury. J Head Trauma Rehabil. 1993; 8(3):86-87. DOI:10.1097/00001199-199309000-00010

9. McCrea M, Guskiewicz KM, Marshall SW, Barr W, Randolph C, Cantu RC, Onate JA, Yang J, Kelley JP. Acute effects and recovery time following concussion in collegiate football players: The NCAA concussion study. JAMA. 2003;290(19):2556-63. [PMID: 14625332] DOI:10.1001/jama.290.19.2556 
10. Iverson GL, Zasler ND, Lange RT. Post-concussive disorder. In: Zasler ND, Katz DI, Zafonte RD, editors. Brain injury medicine: Principles and practice. New York (NY): Demos; 2007. p. 373-403.

11. Defense and Veterans Brain Injury Center working group on the acute management of mild traumatic brain injury in military operational settings: Clinical practice guidelines and recommendations, 22 December 2006. Washington (DC): Defense and Veterans Brain Injury Center; 2006.

12. Hoge CW, Goldberg HM, Castro CA. Care of war veterans with mild traumatic brain injury-Flawed perspectives. N Engl J Med. 2009;360(16):1588-91. [PMID: 19369664] DOI:10.1056/NEJMp0810606

13. Hoge CW, Castro CA, Messer SC, McGurk D, Cotting DI, Koffman RL. Combat duty in Iraq and Afghanistan, mental health problems, and barriers to care. N Engl J Med. 2004; 351(1):13-22. [PMID: 15229303] DOI:10.1056/NEJMoa040603

14. American Psychiatric Association. Diagnostic and statistical manual of mental disorders: DSM-IV. Washington (DC): American Psychiatric Association; 1994.

15. Veterans Health Administration. Implementation of new national clinical reminder, the "Afghan and Iraq postdeployment screen.” VHA Directive 2004-015. Washington (DC): Department of Veterans Affairs; 2004.

16. The Management of Post-Traumatic Stress Working Group [Internet]. Washington (DC): Department of Veterans Affairs; c2004. VA/DOD clinical practice guidelines for the management of post-traumatic stress; 2004 Jan [cited 2009 Apr]; [about 175 screens]. Available from: http://www.healthquality.va.gov/ptsd/ptsd_full.pdf/.

17. Sigford BJ. "To care for him who shall have borne the battle and for his widow and his orphan” (Abraham Lincoln): The Department of Veterans Affairs polytrauma system of care. Arch Phys Med Rehabil. 2008;89(1):160-62. [PMID: 18164348] DOI:10.1016/j.apmr.2007.09.015

18. Veterans Health Administration. Screening and evaluation of possible traumatic brain injury in Operation Enduring Freedom (OEF) and Operation Iraqi Freedom (OIF) veterans. VHA Directive 2007-013. Washington (DC): Department of Veterans Affairs; 2007.

19. The Management of Concussion/mTBI Working Group. VA/DOD clinical practice guideline for management of concussion/mild traumatic brain injury (mTBI). J Rehabil Res Dev. 2009;46(6):CP1-CP68.

20. Beebe J. Rapid assessment process: An introduction. Walnut Creek (CA): AltaMira Press; 2001.

21. Fitch C, Stimson GV, Rhodes T, Poznyak V. Rapid assessment: An international review of diffusion, practice and outcomes in the substance use field. Soc Sci Med. 2004;
59(9):1819-30. [PMID: 15312917]

DOI:10.1016/j.socscimed.2004.02.028

22. Crabtree BF, Miller WL, editors. Doing qualitative research. 2nd ed. Thousand Oaks (CA): Sage Publications; 1999.

23. Miles MB, Huberman AM. Qualitative data analysis. An expanded sourcebook. Thousand Oaks (CA): Sage Publications; 1994.

24. Needle RH, Trotter RT 2nd, Singer M, Bates C, Page JB, Metzer D, Marcelin LH. Rapid assessment of the HIV/ AIDS crisis in racial and ethnic minority communities: An approach for timely community interventions. Am J Public Health. 2003;93(6):970-79. [PMID: 12773364] DOI:10.2105/AJPH.93.6.970

25. Bliese PD, Wright KM, Adler AB, Cabrera O, Castro CA, Hoge CW. Validating the primary care posttraumatic stress disorder screen and the posttraumatic stress disorder checklist with soldiers returning from combat. J Consult Clin Psychol. 2008;76(2):272-81. [PMID: 18377123] DOI:10.1037/0022-006X.76.2.272

26. Batten SV, Pollack SJ. Integrative outpatient treatment for returning service members. J Clin Psychol. 2008;64(8): 928-39. [PMID: 18561182] DOI:10.1002/jclp.20513

27. Soo C, Tate R. Psychological treatment for anxiety in people with traumatic brain injury. Cochrane Database Syst Rev. 2007;3:CD005239. [PMID: 17636792]

28. Bryant RA. Posttraumatic stress disorder, flashbacks, and pseudomemories in closed head injury. J Trauma Stress. 1996;9(3):621-29. [PMID: 8827662]

DOI:10.1002/jts.2490090318

29. Harvey AG, Bryant RA. Two-year prospective evaluation of the relationship between acute stress disorder and posttraumatic stress disorder following mild traumatic brain injury. Am J Psychiatry. 2000;157(4):626-28.

[PMID: 10739425]

DOI:10.1176/appi.ajp.157.4.626

30. Kennedy JE, Jaffee MS, Leskin GA, Stokes JW, Leal FO, Fitzpatrick PJ. Posttraumatic stress disorder and posttraumatic stress disorder-like symptoms and mild traumatic brain injury. J Rehabil Res Dev. 2007;44(7):895-920.

[PMID: 18075948] DOI:10.1682/JRRD.2006.12.0166

31. Levin HS, Brown SA, Song JX, McCauley SR, Boake C, Contant CF, Goodman H, Kotrla KJ. Depression and posttraumatic stress disorder at three months after mild to moderate traumatic brain injury. J Clin Exp Neuropsychol. 2001;23(6):754-69. [PMID: 11910542]

DOI:10.1076/jcen.23.6.754.1021

32. McCauley SR, Boake C, Levin HS, Contant CF, Song JX. Postconcussional disorder following mild to moderate traumatic brain injury: Anxiety, depression, and social support as risk factors and comorbidities. J Clin Exp Neuropsychol. 
2001;23(6):792-808. [PMID: 11910545]

DOI:10.1076/jcen.23.6.792.1016

33. Sbordone RJ, Liter JC. Mild traumatic brain injury does not produce post-traumatic stress disorder. Brain Inj. 1995;9(4): 405-12. [PMID: 7640686]

DOI:10.3109/02699059509005780
34. VA Learning Catalog. Diagnosing mild traumatic brain injury: How do you know? [video]. Washington (DC): Department of Veterans Affairs; 2008. 2 videos.

Submitted for publication January 20, 2009. Accepted in revised form June 9, 2009. 